\title{
Mass Spectrum of the Galactic Center Molecular Clouds
}

\author{
Atsushi Miyazaki \\ Nobeyama Radio Observatory, National Astronomical Observatory, \\ Minamimaki, Minamisaku, Nagano 384-1305, Japan \\ Masato Tsuboi \\ Institute of Astrophysics and Planetary Science, Ibaraki University, \\ 2-1-1 Bunkyou, Mito, Ibaraki 310-8512, Japan
}

\begin{abstract}
We have observed three molecular clumps in the Galactic center region in the CS $J=2-1$ line with the Nobeyama Millimeter Array. We have determined the mass spectrum and other statistical relations for the Galactic center cloud cores. The derived mass spectrum is somewhat steeper than for galactic cloud cores based on molecular line observations. In addition, the spectrum is steeper than that of the large scale Galactic center clumps.
\end{abstract}

\section{Introduction}

The physical characteristics of the molecular clouds in the central region of the Galaxy differ considerably from those in the disk region (e.g., Morris \& Serabyn 1996). The statistical relations, such as the velocity width-radius relation, the LTE mass-virial mass relation, and mass spectrum, provide key information on how stars are formed in such conditions as in this Galactic center region. Previously, we identified a total of 159 molecular clumps based on the CS $J=1-0$ survey data in the Galactic center region with the Nobeyama 45-m radio telescope (Miyazaki \& Tsuboi 2000; Tsuboi et al. 1999; ftp://ftp.nro.nao.ac.jp/users/ tsuboi/GC_CS10/). We determined the statistical relations of these Galactic center clumps. The mass spectral index of the Galactic center clumps in the mass range of $10^{4-6} M_{\odot}$ is approximately equal to that for galactic disk clouds (Miyazaki \& Tsuboi 2000). However, it is the smaller clumps (cores) that are likely to be most relevant to star formation. Therefore, we performed highresolution observations of a few molecular clumps with the Nobeyama Millimeter Array in order to determine the interior structure of the Galactic center clumps.

\section{Observation and Analysis}

We performed observation of 3 molecular clumps, CS0.26+0.02, CS0.76-0.06, and CS1.35+0.26, in the CS $J=2-1$ line with the Nobeyama Millimeter Array (NMA). The size of synthesized beam was about $9^{\prime \prime} \times 4^{\prime \prime}$ in both 3 clumps. We identified cloud cores in the clumps using the "clumpfind program" (Williams et al. 1994). The derived mean parameter of all identified cores is radius, $R=0.12$ $\mathrm{pc}$, velocity width (FWHM), $\Delta V=3.5 \mathrm{~km} \mathrm{~s}^{-1}$, and LTE mass, $M_{\mathrm{LTE}}=38 \mathrm{M}_{\odot}$. The parameter range of the identified cores is roughly equal in each clump. 


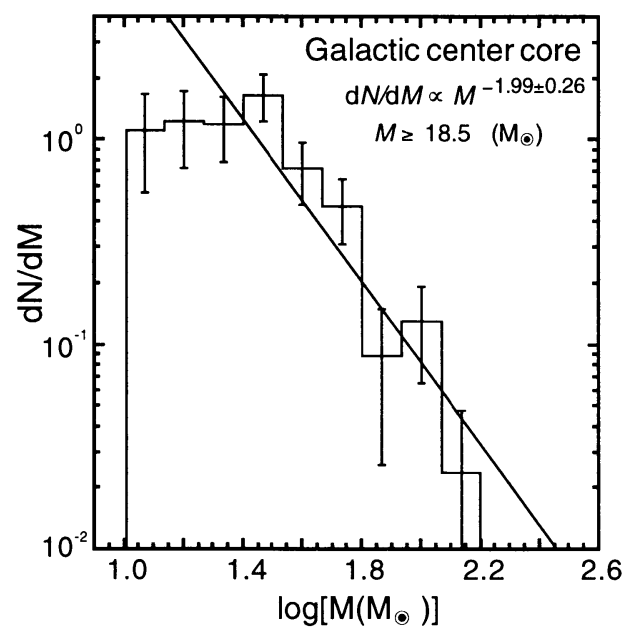

Figure 1. Mass spectrum of the Galactic center cloud cores based on the interferometer observations in CS $J=2-1$ with NMA.

\section{Mass Spectrum}

We constructed the mass spectrum from a sample of 57 cores in the observed 3 clumps, assuming that the spectral index is the same in all 3 regions (figure 1). The mass spectrum of the Galactic center cores (index of -1.99) is somewhat steeper than those of the galactic cloud cores based on the molecular line observations (index of $\sim-1.6$; e.g., Kramer et al. 1998) and that of the large-scale Galactic center molecular clumps (index of -1.59; Miyazaki \& Tsuboi 2000), although the statistical significance is too low to draw firm conclusions as to differences between the Galactic center cores and either galactic disk cores or the large-scale Galactic center clumps.

Recent dust continuum observations of small scale cores in the disk region have given a steeper mass spectrum, which is similar to the Salpeter local IMF (Testi \& Sargent 1998; Motte et al. 1998). These observations, as well as our interferometer observation, must trace dense parts of the cores. Thus our steep spectrum may be due to similar physical processes. On the other hand, one of the open questions about the star formation in the Galactic center region is that the number of massive stars is less than that extrapolated from the disk region (e.g., Morris \& Serabyn 1996). The paucity of massive stars in the Galactic center region may be related to the relatively steep mass spectrum implied by our observations.

\section{References}

Miyazaki, A., \& Tsuboi, M. 2000, ApJ, 536, 357

Morris, M., \& Serabyn, E. 1996, ARA\&A, 34,645

Motte, F., Andre, P., \& Neri, R. 1998, A\&A, 336, 150

Kramer, C., et al. 1998, A\&A, 329, 249

Testi, L., \& Sargent, A.I. 1998, ApJ, 508, L91

Tsuboi, M., Handa, T., \& Ukita, N. 1999, ApJS, 120, 1

Williams, J.P., de Geus, E.J., \& Blitz, L. 1994, ApJ, 428, 639 